\title{
Principales características del comportamiento político electoral en Cartagena de Indias: elecciones de alcalde 2000-2011
}

\author{
Main characteristics of the political electoral behavior in Cartagena de \\ Indias: mayor election 2000-2011
}

\begin{abstract}
RESUMEN
El objetivo de este estudio es presentar los principales aspectos del comportamiento político-electoral de la ciudad de Cartagena de Indias durante la primera década del siglo XXI. En especial, nos enfocaremos en las elecciones a la Alcaldía Mayor de la ciudad. El trabajo avanza en dos partes. En la primera parte se exponen los componentes teóricos de la metodología utilizada.

Aquí se presentan los aspectos más relevantes de enfoque de matriz comparativa y categórica, donde se extraen los aspectos propios y característicos del ciudadano elector cartagenero, con la intención de poder identificar patrones comportamentales que permitan, eventualmente, definir estrategias propositivas para el mejoramiento del ejercicio electoral.

En la segunda parte se presenta un análisis de percepción a partir de la aplicación de una encuesta social a una muestra representativa del censo electoral, con la intención de cotejar el análisis categórico anterior con información emanada de la comunidad votante de la ciudad.

Todo se enmarca en la línea de investigación Democracia y Sociedad Civil del Grupo de Investigaciones de Ciencias Políticas, GIDECP.
\end{abstract}

\section{PALABRAS CLAVE}

Comportamiento Electoral, Participación Política, Elecciones, Cartagena de Indias.

\section{ABSTRACT}

The objective of this study is to present the main aspects of the political-electoral behavior of the city of Cartagena de Indias during the first decade of the 21st century. In particular, we will focus on the mayor elections in the city. This work progresses in two parts. The first part presents the theoretical components of the methodology used.

Here we present the most relevant aspects of a comparative and categorical matrix approach, where the features and characteristics of the voters from Cartagena are extracted, with the intention of being able to identify behavioral patterns that will allow, eventually, to define propositional strategies for the improvement of the electoral exercise.

The second part presents a perception analysis from the application of a social survey to a representative sample of the electoral census, with the intention of comparing the previous categorical analysis with information emanating from the voting community of the city.

Everything is framed in the line of Research Democracy and Civil Society of the Research Group of Political Sciences, GIDECP.

\section{KEYWORDS}

Electoral Behavior, Political Participation, Elections, Cartagena de Indias. 


\section{INTRODUCCIÓN}

El cumplimiento de programas y proyectos propuestos por una Administración Pública en pro del mejoramiento de las condiciones de vida de los habitantes de una sociedad en específico determina su calidad y la prevalencia de corrientes de pensamiento político a lo largo de los años, provocando que algunos gobiernos sean buenos o malos (Navarro, 2005)

Cartagena de Indias es una ciudad con un sistema político muy particular, que la define y le asigna rasgos característicos que incluso se han convertido en tradicionales, dada su prevalencia a través de los años. Hay quienes piensan que se trata de un sistema político propicio solo para las maquinarias de los grandes partidos y de las influencias de la corrupción (Orgulloso, 2005), así como también se piensa que, desde la perspectiva política, las cosas en la ciudad de Cartagena van por buen camino (Cartagena Cómo Vamos [CCV], 2010) y solo existen algunos pequeños retos para hacer de la ciudad un mejor espacio de convivencia ciudadana.

Algo evidente es la falta de estudios rigurosos y científicos que unifiquen criterios y ofrezcan luces objetivas sobre el verdadero espíritu de la política en la ciudad y que permitan, de alguna forma, al ciudadano de común poder reforzar los conocimientos en procura de elegir en forma consciente, obje tiva y acorde a las ideologías y propuestas de los candidatos.
Desde esta perspectiva, el presente artículo nace con la finalidad de poder dar respuesta a diversos interrogantes y necesidades explicitas en cuanto a las expresiones electorales en la ciudad, de tal forma que ello sirva d base para la instauración de sistemas polítcos que contribuyan a un buen ejercicio de la democracia y, lo que es más importante, a la elección de gobernantes que generen desarrollo social en la ciudad.

Por lo tanto, una primera parte del esquema aquí planteado, muestra una revisión analítica de los principales elementos teóricos que giran en torno al concepto inherente de a explicación del comportamiento electora desde una visión de las Ciencias Políticas. Asi mismo, se muestra una breve referencia de la teoría de la selección adversa, para luego establecer los aspectos referidos al marco metodológico sobre el cual se sustenta el desarrollo de la presente investigación.

Atendiendo a que los estudiosos de la ciencia política y una de sus aristas más importantes -la participación política electoracoinciden en afirmar que las personas que se encuentran en los extremos del sistema político, tales como jóvenes o ancianos, pobres o ricos, ilustrados o iletrados, presentan bajo interés en las actividades político-electorales, ya que existen tres grupos de factores que pueden incidir sobre la participación: la posición social del elector, como por ejemplo, el género, la edad, el nivel de estudios, los ingresos y el lugar de residencia; segundo, las actitudes politicas del elector, es decir, el interés, la politización, o la valoración del sistema y las instituciones y, por último, el contexto político de la elección, dentro del que se ubican el sistema electoral, la competitidad electoral o los sistemas de incentivos institucionales (Andauiza \& Bosch, 2004).

Dentro de este esquema consideramos indispensable para la óptica investigativa de presente trabajo, aplicar un instrumento de recolección de datos, a una muestra equivalente al 1,0\% del censo electoral 2011, a efectos de contar con un enfoque empírico $y$ actual que permita interpretar dicho comportamiento.

La parte final está referida principalmente los resultados encontrados y a la determinación de algunas conclusiones y recomendaciones que servirán de base para estudios posteriores que se realicen, siguiendo la línea de investigación en el marco de los sistemas políticos.

\section{Una aproximación teórica}

\subsection{Rasgos básicos de la participación po-} lítica

Antes de exponer la conceptualización sobre los diferentes enfoques explicativos del comportamiento electoral, se hace necesario determinar sus rasgos teorréticos. Según la mayoría de los teóricos de las Ciencias Políticas actuales, la participación en la toma de decisiones es uno de los papeles centrales que realizan los ciudadanos en la democracia. En a toma de dichas decisiones aparecen con un rol preponderante cuestiones como: qué candidato elegir o qué partido apoyar (Anduiza \& Bosch, 2004).

En esta línea un elemento de cuidadoso análisis por parte de las Ciencias Políticas es el llamado comportamiento político de los electores. Anduiza y Bosch (2004) señalan dicho comportamiento como "la manera en que los ciudadanos se conducen en sus relaciones con el sistema político" (p.65).

Esta "manera de conducirse los ciudadanos" con la estructura sociopolítica puede ser analizada a partir de cuatro preguntas fundamentales: (I) ¿De qué manera se participa?, (II) ¿Quién participa?, (III) ¿Por qué se participa?, y, (IV) ¿Qué consecuencias tiene la participación?

En cuanto al primer interrogante (¿De qué manera se participa?), encontramos que la participación política se materializa en diferentes formas que van desde el voto, al activismo en asociaciones $u$ organizaciones.

La intensidad y frecuencia de las formas de participación son variables y oscilan desde el voto individual a la participación corporativa en pro de muchos votos. Aquí se subraya como elemento de análisis el repertorio y la frecuencia o intensidad de la participación (Anduiza \& Bosch, 2004). El repertorio alude a la variedad de formas de participación política que un ciudadano utiliza en un determinado tiempo y la frecuencia hace referencia número de veces en que se ha ejercido una determinada acción. 
Verba y Nie (1972) afirman que los ciudada- Además de estas clasificaciones del tipo de nos suelen "especializarse" en el uso de dis- participación política se puede mencionar la tintas formas de participación política.

distinción entre la participación electoral y no electoral (Anduiza \& Bosch, 2004).

En lo relativo al segundo interrogante

(¿Quién participa?) según Eva Andauiza y La primera es una forma de participación Agustí Bosch (2004, citando a Milbrah, 1977) que se inserta dentro de las formas o mase pueden distinguir en la participación polí- neras convencionales de participación en tica de los ciudadanos tres grupos: los Apá- las democracias representativas dentro de ticos "aquellos que no participan ni se inte- los procesos electorales (Anduiza \& Bosch, resan en política"; los Espectadores "quienes 2004, p.28) de manera genérica el término se limitan a contemplar el devenir de la polí- " "Participación Electoral" se usa como sinótica y que participan esporádicamente", y los nimo de voto. La segunda forma de partiGladiadores "aquellos que participan activa- cipación no electoral alude a las formas de mente" (Anduiza \& Bosch, 2004, p.31).

Otra de las taxonomías en que se puede clasificar la participación política es la presentada por Barnes y Kaase (1979, citada por Anduiza \& Bosch, 2004) que distingue dos tipos de participación a saber: Primero, los Inactivos que no participan en nada a nivel político ni se identifican con ningún partido. Segundo, los Conformistas aquellos que solo participan de manera convencional pero que

no participan en acciones de protesta. Estos últimos se subdividen en tres categorías, es decir, los Contestatarios "que rechazan la participación convencional y se limitan a la participación no convencional tanto legal como ilegal"; los Reformistas "quienes participan en formas convencionales y alguna formas legales de protesta" $y$, finalmente, los Activistas, aquí se agrupan todas las formas de particípación política, tanto convencionales como no convencionales e incluso ilegales (Anduiza \& Bosch, 2004, p.34) cipación no electoral alude a las formas de
participación no inscritas en lo tradicional. Por su parte, la participación convencional la no convencional "distingue las formas de participación política en tanto si se ajustan o no a las normas sociales y valores de una sociedad". La participación convencional (ta como votar, participar en campañas, actividades cooperativas de ámbito local, contactos con políticos) posee estatus legal y la no convencional (tal como boicots, objeción de conciencia e insumisión, bloqueos de tráfico) n muchos casos es extralegal.

Por último, otra distinción relevante es la presentada entre la participación basada en Mientras que la "salida" alude a posibilidad de ejercer presión como por ejemplo abandonar un determinado partido o no votar, la "voz" hace referencia al acto de comunica de hacer explícito el descontento hacia un sistema político mediante protestas públicas (Anduiza \& Bosch, 2004, p.29) la voz y la participación basada en la salida.
En lo referente al tercer interrogante (¿por qué se participa?) según los autores anteriormente citados, entre ellos, Anduiza \& Bosch (2004), sostienen que entre los diferentes factores que condicionan y pueden determinar la participación política de los ciudadanos tenemos: (I) Los recursos individuales donde encontramos variables como la edad nivel de escolaridad, nivel de ingresos, tipo de ocupación y tiempo disponible posibilitan una mayor o menor participación; (II) las redes sociales, grupos y organizaciones, donde el contacto con personas y organizaciones puede servir de motor en la participación política; (III) el interés y compromiso por la política que genera en los individuos y en los grupos nuevas percepciones de lo político que puede incidir en la toma de decisiones $y$, (IV) el contexto político e institucional en sociedades interconectadas; el contexto político local e internacional condiciona la toma de decisiones e inciden en el tipo de participación política que se ejerza (Anduiza \& Bosch, 2004, p.19).

1.2. Tesis principales de los enfoques analítcos del comportamiento electoral

Son múltiples las respuestas que pueden obtenerse al estudiar el comportamient político electoral. Sin embargo, de manera general las Ciencias Políticas plantean diversas concepciones teóricas que tienen por finalidad explicar las particularidades de dicho comportamiento. Además la explicación sobre el voto, el elemento básico del comportamiento electoral necesariamente incluye, una combinación de variables cuyo impacto puede cambiar de una elección a otra. Veamos los aspectos fundamentales de estas teorizaciones:

Una mirada a las teorías contemporáneas sobre comportamiento electoral, ha de tomar en cuenta, en primer lugar, la llamada tradición sociológica o modelo sociológico, presente en el libro de Paul Lazarsfeld, The Peoples Choice (1994) que hace énfasis en el impacto del proceso familiar de socialización de los ciudadanos (una proporción alta de los votantes adopta la misma identificación partidista de sus padres), el sitio de trabay el vecindario. Siguiendo esta tradición otros autores han formulado explicaciones que incorporan el contexto de las redes sociales y los modelos de influencia que impactan el voto (Huckfeldt, 1983, p.651). Uno de ellos es el llamado modelo de Columbia, que describe la influencia de la socialización en la toma de decisión sobre si votar o no, y a quién votar; de esta forma resalta la importancia de la integración social como elemento motivador de la participación política.

Dentro de este contexto, se afirma por ejemplo que los electores casados y con una socialización política importante, participaban más que los solteros o con un menor grado de socialización política. De igual forma, se enfatiza en la importancia de los grupos primarios a la hora de compartir y transmitir una serie de valores y percepciones de la realidad política, llegando a afirmar que los miembros de un mismo grupo primario tienen el mismo voto y están afiliados al mismo partido. 
Además en cuanto a aquellos que están indecisos a la hora de votar, según esta perspectiva suelen hacerlo siguiendo a sus personas cercanas. Actualmente, la integración socia es considerada como un indicador de capital social, en ese sentido podría decirse que los medios masivos de comunicación surten importantes efectos en las campañas políticoelectorales, mas no es esta a la conclusión a la que ha llegado la escuela de Columbia, ya que afirma, que los electores deciden según el grupo al cual pertenecen.

El modelo define al voto como un acto de afirmación, poniendo énfasis en la proporción de votos que los diferentes grupos de ciudadanos asignan a un determinado partido político (Lazarsfeld, Berelson, 1994). Las diferencias existentes entre los patrones electorales de los grupos se relacionan con su posición en la sociedad, es decir, su ubicación dentro de la estructura social, y cómo esto determina si participa o no y cómo su posición ha producido vínculos con determinados partidos.

Este enfoque supone por tanto, que los individuos tienen un grado de control sobre la composición política y social de su entorno y es por eso que las redes sociales aparecen como un resultado de la interacción entre el contexto social externo y las preferencias individuales (Carmines \& Huckeldt, 1996, p.233). El votante escoge, asi, fuentes de información del contexto según sus propias preferencias, obviamente incorporando los propios sesgos de dicho contexto. A diferencia de modelos anteriores de esta tradición tal perspectiva incluye la volición individua $y$, por consecuencia, evita el determinismo sociológico de los modelos precedentes.

Otra de las teorías que explican el comportamiento electoral se desarrolló en la Universidad de Michigan, expuesta en un libro titulado The American Voter (Campbell, Miller, Converse \& Stokes, 1960). Aquí los autores construyen un modelo que explica una alt proporción del voto de los norteamericanos en la elección presidencial de 1956 con base en el famoso Funnel (embudo) que básicamente depende de dos variables: la identificación partidista y los temas de campaña. El Centro de Investigaciones sobre Opinión Pública de la Universidad de Michigan sigue utilizando este modelo, pero ha perdido su poder explicativo inicial a causa del ocaso de fenómeno de identificación partidista, de surgimiento de un electorado más independiente que vota sobre temas y por las características personales de los candidatos.

Por ello, estudios posteriores examinan más profundamente el impacto de la percepción de los candidatos sobre el voto (Converse \& Markus, 1979). El voto para este modelo es considerado como un mecanismo de afimación, pero no de pertenencia a una clase social, sino que es un proceso de largo plazo que ha llevado al individuo a identificarse con un partido en particular. Se identifican aquí las actitudes políticas como el factor determinante en la decisión de participar no en una convocatoria electoral, profundizando en las características psicológicas de percepción del votante ante diferentes elementos del sistema político y enmarcándolo en un rol participativo e influyente en política. Sobre todo, se hacía hincapié en la identificación partidista como elemento explicativo clave del comportamiento de los electores. El énfasis del modelo es el individualismo, porque considera a la person como un ente individual que desarrolla una identificación psicológica duradera con un partido a través de un proceso de socialización (Campbell \& Converse, 1960). La identificación hacia un partido se adquiere inicialmente por medio de la familia, reforzándose por el ingreso posterior a ciertos grupos, lo que puede generar la permanencia de voto en dicho grupo.

Las actitudes políticas tienen un efecto en el Desde 1957, con la publicación de Downs, se comportamiento y la participación electoral ya que pueden involucrarse aspectos afectivos, cognitivos y evaluativos, que podrían predisponer al individuo a actuar en una dirección u otra, y con un grado de intensidad diferente. Así, aspectos de conocimiento político, identificación ideológica o confianza en las instituciones e incluso la tradición familiar, podrían incidir en determinado comportamiento electoral. Estas identificaciones suponen el reflejo de conflictos socioestructurales, como aspectos de clase y posiciones religiosas, de ahí que en los análisis en los que esta característica se tiene en cuenta aparezca como el factor más importante, por delante de aspectos económicos y sociales (Lancaster \& Lewis-Beck, 1986).

La tercera tradición teórica se ubica en la literatura fundamentada en la llamada elección racional. La obra representativa de esta perspectiva sobre el comportamiento electoral se encuentra en el libro de Anthony Downs, An Economic Theory of Democracy (1957). En ella, el autor formula un modelo capaz de predecir cómo un ciudadano hace un cálcuo en torno a la decisión de votar o no votar con base en los costos personales que representa hacerlo. Cuando la decisión es votar, se haría con base en una decisión racional que maximizaría sus intereses. El modelo postuque tanto los partidos políticos como los candidatos estructurarán un programa de gobierno y el votante escogerá el partido y/o candidato que más se aproxime a sus intereses personales. han obtenido importantes avances en los estudios sobre elección racional convirtiéndocomportamiento electoral. El modelo se ha como el pocketbook voting, que se refiere a las decisiones de los votantes en términos del estado actual de su bolsillo: Si el votante está bien económicamente, votará por el partido o candidato que él percibe que continuará as políticas que le han beneficiado; si no se encuentra bien económicamente, votará por el partido o candidato de la oposición. En ese orden de ideas, los individuos no votan atendiendo a su participación en un grupo o clase social; sino por las percepciones individuales o colectivas que tienen del grupo que ostenta el poder, frente a los partidos opositores y su capacidad de brindar credibilidad para el cumplimiento de las promesas de campaña. se en una forma corriente de interpretar el refinado bastante, desarrollando conceptos 
Aspectos como la clase social o la identifi- economía, lo que se llama sociotropic voting cación partidaria constituyen elementos de (Kinder \& Kiewiet, 1981). Si su percepción de influencia en el largo plazo en el votante y futuro es muy optimista, es muy probable por ende, le otorga estabilidad en el voto por que voten por el partido o el candidato que ese partido. Pero, al momento en que las di- se encuentra manejando el gobierno actual. visiones de clases dejan de ser prioridad para determinar la conducta (Clark, Lipset, 1991; La literatura que cae dentro de esta tradición Franklin, Mackie, 1992), o cuando los niveles ha experimentado, sin embargo, transforde identificación partidaria decrecen (Dal- maciones significativas. Fiorna (1976) poston, Flanagan, 1984; Crewe, Denver, 1985), tula un modelo teórico híbrido para explicar son factores de corto plazo que se convierten la decisión de voto. Más que la perspectiva en determinantes del votante.

En este sentido, el votante, sobre la base de una conducta racional, apoyará al candidato que más se acerque a sus intereses, es decir, el elector reconoce su interés personal y realiza una evaluación de cada candidato adaptándose a sus propios intereses, los cuales ya están previamente definidos.

Existe otra tendencia semejante a la del pocketbook voting, pero es más amplia: la votación retrospectiva (retrospective voting). Este enfoque postula que los votantes toman su decisión sobre la base de una evaluación del anterior gobierno (Florina, 1981). Si creen que el gobierno anterior tuvo un buen desempeño, votarán por el partido y el candidato que representa el continuismo; si, por el contrario, consideran que el gobierno anterior fue pésimo, votarán por el candidato o candidatos de la oposición.

Finalmente hay otra variante de escogencia racional que postula que los ciudadanos votan siguiendo los criterios de bolsillo, sino sobre la base de su percepción futura de la económica (instrumental), incorporando una perspectiva psicológica. Este modelo es una reformulación de la proposición de Rier y Ordeshook (1968) donde los votantes no toman sus decisiones de voto, solament de costos y beneficios), sino también a partir de los deseos de los votantes que expresa solidaridad de clase, identificación partidista, o simplemente un deber cívico (García \& Holguín, 2002, p.10). En suma, la perspectiva instrumental ha incorporado con el tiempo factores de expresión social y afectiva en sus análisis de las decisiones de voto.

El marco teórico de este estudio se posa dentro de la tradición de elección racional, siguiendo concretamente las orientaciones teóricas y metodológicas del libro, Reasoning and Choice: Explorations in Political Psychology (Sinderman, Brody \& Tetlock, 1991). La pregunta fundamental de este libro recoge una problemática que es un paradigma en la literatura sobre la democracia y las elecciones: ¿Cómo es posible que los ciudadanos tomen una decisión de voto racionalmente cuando muchos no tienen el deseo, la eneren una perspectiva instrumental (un cálculo gía y el tiempo para informarse sobre candidatos, partidos políticos y políticas públicas?

De la consideración de que la mayoría de los votantes potenciales no son ciudadanos modelos en términos de estar bien informados, o de participar activamente en la política, es que frecuentemente se deduce que es imposible para la mayoría de los votantes depositar un voto a base de raciocinio. Como consecuencia, el votante racional se limita aquellos individuos que tienen un conocimiento amplio de las políticas públicas, los temas de campaña y las características personales de los candidatos que se supone son necesarios para llegar a una decisión sobre quién representa mejor los intereses personales (voto instrumental). En resumen, este postulado sobre la incapacidad de la mayoría de los votantes de votar racionalmente, socava la capacidad de la literatura de escogencia racional de pronosticar el voto.

Siguiendo la idea de satisficing de Herbert Simon (1957 y 1985), Sinderman, Brody y Tetlock sostienen que, aun con falta de información, los votantes pueden comportarse racionalmente cuando depositan sus votos: ellos utilizan heurísticas o atajos para tomar ecisiones que no son menos racionales que las de aquellos ciudadanos bien informados políticamente. La mayoría de los estudios electorales mediante encuestas, no distinguen entre los votantes que cumplen con los requisitos de ciudadanos bien informados y los que no son, por eso Sinderman, y sus colegas, argumentan que un modelo tan exclusivo no es apropiado para explicar deci- siones de voto, puesto que los votantes utilizan cadenas de racionamiento distintas en el proceso de hacer una decisión electoral.

Los "votantes sofisticados políticamente" llegan a una decisión de voto sobre una previa cadena extendida de racionamiento, que incluye bastante información y la posibilidad de manipular sus conocimientos en una forma en que es factible maximizar las preferencias. Los "menos sofisticados" no utilizan una cadena tan elaborada y basan sus decisiones de voto en una heurística, tal como la preferencia por un candidato o identificación partidista.

En contraste con buena parte de la literatura dentro de la tradición racional, que enfatiza el papel de lo afectivo sobre lo cognitivo en los votantes menos sofisticados, Sinderman arguye que las decisiones de voto para los sofisticados y menos sofisticados se basan en una combinación de lo afectivo y lo cognitivo (Sniderman, Brody, Tetlock, 1991, p.21). Tomando las consideraciones teóricas anteriores, en el presente estudio se desarrollarán modelos diferentes para explicar el comportamiento electoral según el nivel de sofisticación de los votantes.

Desde esta perspectiva, se desarrollarán unos modelos de asociación multivariable para averiguar qué variables independientes afectan más fuertemente la decisión de voto-identificación partidista, características de los candidatos, preferencias de políticas públicas, orientaciones ideológicas. Particularmente se plantea como hipotesis que los votantes más sofisticados utilizan una cade- 
na de racionamiento más elaborada que los menos sofisticados. Por ejemplo: que los más sofisticados utilizan no solamente más factores en la decisión del voto, sino también que den más peso a ciertas variables que otras.

El sofisticado puede enfatizar una combinación que incluirá un análisis de las postulaciones de los candidatos sobre los asuntos de la campaña, una evaluación de las capacidades de los candidatos y tal vez, la afiliación partidista. Los menos sofisticados, a su turno, pueden utilizar atajos para decidir su voto, tal vez, solamente afiliación partidista y/o favorabilidad hacia los candidatos. La tesis de Sinderman, Brody y Tetlock es que las decisiones de voto de los menos sofisticados que usan atajos, en vez de una cadena extendida de razonamiento, no es menos racional que la de los sofisticados.

En resumen, las teorías antes expuestas, incluyen en grados distintos las siguientes variables, que podrán ser de utilidad para predecir el comportamiento electoral: el impacto del proceso de socialización política, la identificación partidista, la situación económica del votante y su percepción del futuro, el desempeño del gobierno en el poder, el desarrollo de la campaña y las característica de los candidatos. En su conjunto, estas variables determinan el comportamiento político electoral de los votantes.

1.3. Teoría de la selección adversa en el ámbito de la política y sus determinantes en el comportamiento político electoral

En la literatura de las Ciencias Económicas existe la denominada teoría de la selección adversa, la cual en algunos casos sirve para explicar no solo fenómenos estrictamente económicos sino también algunos problemas derivados de fenómenos sociales complejos. Para el caso que compete directament esta investigación, se busca fundamentar e porqué algunos enfoques teóricos de esta teoría podrían ser útiles para entender las características propias del entorno político de la ciudad de Cartagena.

La realidad que percibe cualquier ciudadano sobre las "circunstancias electorales" en esta ciudad, podría arbitrariamente arrojar conclusiones sobre el "infortunio" en cuanto la elección de sus gobernantes, ajeno al proceso presentado por otras ciudades del país que han contado con la "fortuna" de elegi alcaldes y gobernadores que lideran el desarrollo de sus comunidades y sobre las cuales se ejerce un cambio significativo a nivel de satisfacción de necesidades básicas de sus habitantes.

Dentro de este contexto, se pretende aplica los fundamentos teóricos de la selección adversa, a efectos de determinar si se dan los supuestos que permitan concluir, entre otros aspectos, si el comportamiento político electoral de los votantes de la ciudad de Cartagena para la elección de alcalde se enmarca con este esquema.

La selección adversa es un problema de mercado que nace principalmente por la falta de información o información asimétrica exis- tente en los mercados competitivos de bienes y servicios. En este sentido, el consum dor o usuario final de un bien no puede tener en la mayoría de los casos información precisa sobre la calidad del bien, solo hasta que ha adquirido y por tanto, siempre existe disyuntiva sobre pagar la cantidad suficiente de dinero por un bien sobre el que no se tiene la certeza absoluta sobre sus condiciones y calidad (Montoya \& Montoya, 2005).

Por tanto, ante la existencia en el mercado de bienes de buena y mala calidad y ante la disyuntiva del consumidor sobre el precio a pagar, es de esperarse que la demanda total se reduzca a tal punto que el precio que prevalecerá en el mercado será solo el que están dispuestos a pagar los consumidores a los que les es indiferente la selección de un bien de mala calidad (Akerlof, 1970). Ello explica el porqué en el mercado predominan os bienes y servicios de mala calidad aun ante la complejidad de los sistemas productivos modernos.

Este mismo racionamiento emanado de teoría de la selección adversa ha sido utilizado en diversas investigaciones de ámbitos diferentes, por lo que será aplicada para explicar el comportamiento electoral y ayudar determinar las causas por las cuales son escogidos los gobernantes en una sociedad específica.

De esta manera, si se acepta el supuesto que En el desarrollo de esta investigación aplicael sistema político es como un mercado en mos un instrumento de medición, compuesdonde los votantes son los demandantes y to de 24 preguntas, que nos suministraron

los candidatos los oferentes, siendo el voto el precio que tienen que pagar los unos por adquirir el servicio ofrecido por los otros, entonces la teoría de selección adversa aplica eficientemente puesto que en dicho mercatambién existe asimetría en la información en la mayoría de los casos.

Si los votantes tienen una duda sobre qué candidatos son de buena o mala calidad, es probable que muchos de ellos se rehúsen a votar, ocasionando un descenso en la cantidad total de votantes y dejando únicamente en el mercado a aquellos a los que les es indiferente elegir o no, gobernantes de buena calidad (Navarro, 2005).

El desarrollo de la teoría de la selección adversa con una visión del sistema político, debe ser probado en distintas sociedades a fin de comprobar su correspondencia empírica y poder generalizar sobre su aplicabilidad para este ámbito específico de las Ciencias Sociales.

En estas circunstancias, para la presente investigación se ha utilizado información proveniente de fuentes primarias, de tal forma que se indagó sobre los aspectos más subjetivos y característicos de los votantes, aplicando un esquema interpretativo, el cual se argumenta en forma consistente con la teoría.

REVISTA VIS IURIS I No. 3, Vol. 2185 - 109 I Enero-Unio, 2015 I Universidad Sergio Arboleda I Esculela de Derecho I Seccional Santa Marta, Sede Centro 
información primaria que permitiera medir te, se evalúa el nivel de votación y de corresentre otros aspectos, la edad, el nivel de es- pondencia teórica para interpretar el comcolaridad, la percepción que se tiene sobre portamiento político electoral de la ciudad de las instituciones públicas, la motivación a la Cartagena y su correspondencia con la aplihora de depositar el voto y la filiación par- cación de la teoría de la selección adversa. tidista, así como también indagar sobre los aspectos y tópicos relacionados con su preferencia electoral. Este instrumento se aplicó a 384 hombres y mujeres, ciudadanos en ejercicio, inscritos para votar, habitantes de los diferentes barrios de Cartagena.

Para hacer la distribución por localidades se tomaron como base las estadísticas arrojadas por el censo electoral para elección de autoridades locales de la Registraduría $\mathrm{Na}$ cional del Estado Civil en el proceso electoral 2011, y de allí, según la distribución obtenida por localidades, se realizó la distribución porcentual para hombres y mujeres, por barrio, ubicando consecuentemente el estrato.

El muestreo estadístico para la selección de las unidades de estudio, se realizó a partir de la implementación de la fórmula estadística para la determinación de una muestra de poblaciones finitas o conocidas, con un margen de error del $10 \%$.

De la aplicación del instrumento y su posterior procesamiento, se extrajeron los principales indicadores descriptivos, creando estadísticos descriptivos, calculando algunas medidas de tendencia central que nos permitieron un análisis cabal y consistente de la información obtenida.

Del análisis estadístico descriptivo resultan-
Del análisis de corte cualitativo y categórico, luego de la construcción de una matriz de datos comparativos y propios de cada un de las administraciones públicas de los primeros 10 años del siglo XXI en la ciudad de Cartagena, se tuvieron en cuenta los aspectos característicos, fortalezas, debilidades, propuestas y otros.

Aspectos sociodemográficos del elector cartagenero

El presente apartado mostrará un panorama general basado en la interpretación de los resultados obtenidos con el procesamiento y aplicación de la base de datos resultante de la encuesta aplicada a 384 personas mayores de edad, cuya característica hace referencia a lo observado durante el comportamiento electoral de los encuestados.

Cabe señalar que es posible encontrar algunas pequeñas diferencias con respecto a la distribución propuesta metodológicamente, puesto que al realizar el trabajo de campo las unidades muestrales disponibles por barrios tendieron a cambiar con respecto a lo esperado en la distribución propuesta.

\section{Caracteristicas del comportamiento elec-}

Se presenta inicialmente la distribución por sexo, obteniendo la gráfica que muestra esa información.

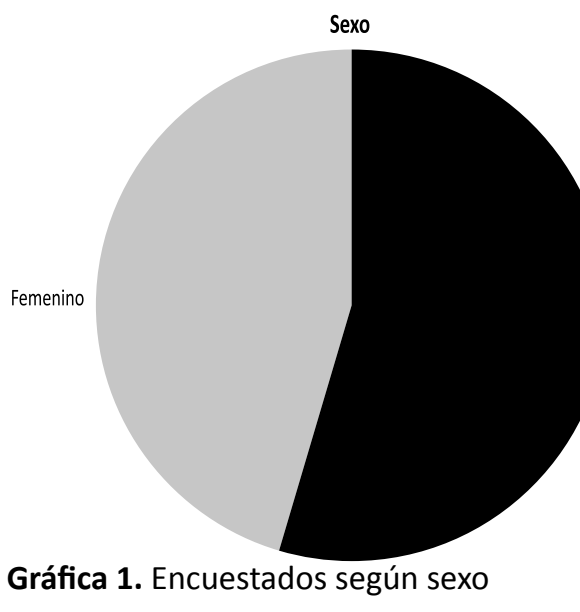

Se observa que 207 encuestados pertenecen al sexo masculino, representando el $53 \%$ de total de las personas que fueron abordadas para aplicarles el formato de captura de la información. Esta cifra se determinó por el resultado arrojado del censo electoral 2011.

En esa misma medida, al cruzar el sexo de as personas encuestadas con los rangos etarios establecidos en el formato de captura, e muestra la distribución de frecuencias de total encuestado, teniendo en cuenta el sexo $y$ rango de edad al que pertenecen. En ese sentido, se observa que de las 207 personas de sexo masculino encuestadas, la mayoría (45\%) tienen edades de 40 años o más. Las personas con edades entre 35 y 40 años, representan el segundo rango etario, tal $y$ como se indica a continuación

Tabla 1. Edad-SeXo
\begin{tabular}{|c|c|c|c|}
\hline RANGO DE EDAD & \multicolumn{2}{|c|}{ SEXO } & TOTAL \\
\hline & Masculino & Femenino & \\
$18-24$ & 25 & 32 & 57 \\
$25-29$ & 22 & 35 & 57 \\
$30-34$ & 30 & 30 & 60 \\
$35-40$ & 36 & 32 & 68 \\
40 o más & 94 & 48 & 142 \\
\hline TOTAL & 207 & 177 & 384 \\
\hline
\end{tabular}
Fuente: Del autor tomada de encuestas aplicadas

Era de especial interés para alcanzar los objetivos perseguidos por este estudio, que las personas a encuestar fueran mayores de edad, ciudadanos en ejercicio y que se encontraran inscritas en el censo electoral. En este orden, se procedió a aplicar el instrumento de captura de la información según la distribución por edad, sexo y barrio obtenida del aplicativo MIDAS de la Alcaldía Mayor de Cartagena de Indias.

Teniendo en cuenta el sexo y el rango de edad, se obtiene que el $95 \%$ de los encuestados está inscrito para votar en la ciudad. El rango de los inscritos suele seguir el mismo patrón en cuanto a las edades y sexos, en tanto a que, como ya se afirmó, la mayoría de estos tienen más de 40 años y son del sexo masculino. Sin embargo, es importante destacar que existe una alta prevalencia de personas con edades de entre 18 y 24 años de edad, inscritas en el censo electoral de la ciudad de Cartagena, las cuales son en su mayoría mujeres.

En cuanto al nivel de escolaridad se observa que la mayoría de personas (116), afirma tener el grado de bachiller, mientras que la segunda categoría de mayor registro fue la 
de profesional. Ello muestra que gran parte Además, de este 31 \% la mayoría son ciudel censo electoral cartagenero posee el nivel básico para votar. Ver Gráfica 2

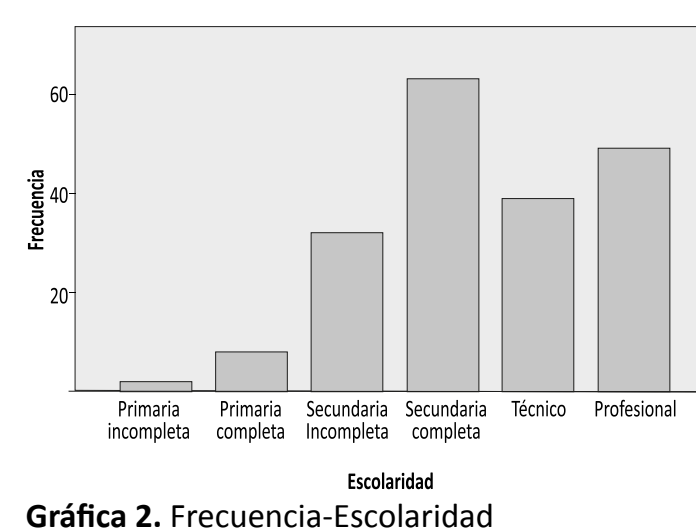

Fuente: Del autor tomada de encuestas aplicados

Para complementar un poco más la descripción sociodemográfica, se buscaba conocer, a partir de los lugares de residencia de las personas encuestadas, su estrato socioeconómico para realizar un análisis que permitiera deducir cuál es el estrato preponderante, además de conocer el que posee la mayor cantidad de personas con mayor o menor nivel de escolaridad.

Se utilizó como apoyo principal la base de datos de la Secretaría de Planeación Distrita de Cartagena, en la cual se calcula un estrato promedio modal* para cada barrio registrado, cotejado con los barrios resultantes de la aplicación del instrumento de captura utilizado. Por lo tanto, al realizar la contingencia entre estrato promedio y escolaridad se obtuvo que entre los encuestados, el estrato promedio preponderante es el estrato 2 , el cual reporta un total de 119 personas del total de encuestados, que representan el $31 \%$ del total.

\section{Muestra el promedio de estrato de un barrio, teniendo
en cuenta cuál de los estratos es el que más se repite} dentro de la delimitación geográfica de cada barrio. dadanos con un nivel de escolaridad de secundaria completa. En el nivel profesional se registran 77 ciudadanos ubicados en los estratos 1,2 y 3 , frente a un escaso $10 \%$ (35) que pertenece a los estratos 4,5 y 6 ; lo que indica que entre los estratos más bajos se encuentran personas con el nivel básico de escolaridad para afrontar procesos electorales, pudiendo propiciar una elección mucho más objetiva y consciente.

Sin embargo, como se indica más adelante se pudo observar, según pregunta formulada en la encuesta, que las personas no tienen mucho interés en la política, e incluso esta se refleja en cuanto a los procesos y asuntos políticos locales. La Tabla 2 indica lo señalado sobre el nivel de escolaridad frente al estrato socioeconómico.

\subsection{Aspectos político-electorales: Lo que} piensa el elector

La caracterización del comportamiento politico electoral de los cartageneros también incluye observar las orientaciones del electo hacia los objetivos políticos, en este caso, hacia las instituciones de la democracia, a efectos de interpretar algunos comportamientos tendencias propios de la población encuestada que podrían permitir obtener respuestas sobre el comportamiento electoral y la teoría de la selección adversa, además de conocer los rasgos propios de las personas de ejercer su derecho cuando hay debate electoral.
Tabla 2. Escolaridad-Estrato

\begin{tabular}{|l|c|c|c|c|c|c|c|c|}
\hline \multirow{2}{*}{ ESCOLARIDAD } & \multicolumn{7}{|c|}{ ESTRATO } & \multirow{2}{*}{ TOTAL } \\
\cline { 2 - 8 } & $\mathbf{1}$ & $\mathbf{2}$ & $\mathbf{3}$ & $\mathbf{4}$ & $\mathbf{5}$ & $\mathbf{6}$ & SD & \\
\hline Primariaincompleta & 5 & 4 & 0 & 0 & 0 & 0 & 2 & 11 \\
\hline Primaria completa & 7 & 2 & 1 & 0 & 0 & 0 & 3 & 13 \\
\hline Secundariaincompleta & 15 & 17 & 9 & 1 & 0 & 0 & 7 & 49 \\
\hline Secundaria completa & 36 & 35 & 22 & 3 & 0 & 3 & 17 & 116 \\
\hline Técnico & 21 & 28 & 11 & 2 & 4 & 0 & 18 & 84 \\
\hline Profesional & 12 & 33 & 32 & 4 & 12 & 6 & 11 & 110 \\
\hline Otros & 0 & 0 & 0 & 0 & 0 & 0 & 1 & 1 \\
\hline TOTAL & 96 & 119 & 75 & 10 & 16 & 9 & 59 & 384 \\
\hline
\end{tabular}

Fuente: Del autor tomada de encuestas aplicadas

Frente a este tópico los cartageneros respondieron a la pregunta de su interés por la po-

representa los aspectos sumados de mucho bastante, no alcanza a generar procesos de lítica, un nada despreciable $43 \%$ de poco in- participación interesantes que provoquen terés por este aspecto. Se observa en la tabla intervención efectiva de los cartageneros en siguiente, que el porcentaje más alto corres- los procesos electorales y sus diferentes arisponde al poco interés por la política, es de- tas.

cir, la mayoría de los encuestados manifiesta

que los procesos y aspectos relacionados Este resultado corresponde con la clasificacon el ejercicio de la política en el entorno ción clásica (Milbrath \& Goel, 1977), que dislocal, les interesa muy poco. Este porcentaje tingue tres grupos de ciudadanos según sus es aún más preocupante si se suma el obte- niveles de participación política entre apátinido en el aspecto de ningún interés por este cos, que para nuestro caso aplica al 67,5\% tópico, obteniendo un porcentaje del $67,5 \%$ aproximadamente correspondiendo a aquede carencia de interés; frente al $30,5 \%$ que llas personas que no participan activamente

Tabla 3. Válidos-Porcentaje

\begin{tabular}{|l|r|r|r|c|}
\hline \multicolumn{1}{|c|}{ VálLDOS } & FRECUENCIA & PORCENTAE & PORCENTAEVÁLIDO & $\begin{array}{r}\text { PORCENTAE } \\
\text { ACUMULADO }\end{array}$ \\
\hline Mucho & 58 & 15,1 & 15,1 & 15,1 \\
\hline Bastante & 59 & 15,4 & 15,4 & 30,5 \\
\hline Poco & 165 & 43 & 43,0 & 73,4 \\
\hline Ninguno & 94 & 24,5 & 24,5 & 97,9 \\
\hline No sabe & 4 & 1,0 & 1,0 & 99,0 \\
\hline No responde & 2 & 0,5 & 0,5 & 99,5 \\
\hline Novalida & 2 & 0,5 & 0,5 & 100,0 \\
\hline Total & 384 & 100,0 & 100,0 & \\
\hline
\end{tabular}

Fuente: Del autor tomada de encuestas aplicadas 
ni se interesan por la política; un 15,1 \% co- muy alta percepción sobre el bajo conocirresponde a espectadores, que son aquellos miento de comunidad y de las necesidades que contemplan la política desde la barrera, de la población de sus gobernantes, ya que con participación esporádica y los gladiado- la mayoría piensa que los políticos no se inres que corresponden al $15,4 \%$ y que tienen teresan por lo que puedan pensar y sentir los una participación activa, frecuente y regular. ciudadanos del común.

Este porcentaje podría reflejarse en un frac-

cionamiento o división en la toma de decisio- A la pregunta realizada sobre si comparten nes de interés para la ciudad.

Los analistas coinciden en afirmar que el in terés de los ciudadanos por la política estimula y favorece la participación electoral y que por tanto, la ausencia de interés indica

un distanciamiento entre el mundo de la política y los electores (Andauiza \& Bosch, 2004, p.128).

En esa medida y tal y como se muestra en la siguiente tabla de caracterización, al ser consultados para que expresaran su opinión en cuanto a la afirmación: "los políticos no se preocupan por lo que piensa la gente como yo", un representativo $78,1 \%$ (303) de las personas encuestadas manifiesta estar muy En cuanto a la afirmación: "En el distrito de de acuerdo o de acuerdo con tal afirmación; Cartagena, esté quien esté en el poder, siemlo que refleja que las personas tienen una pre busca sus intereses personales", se obtie-

Tabla 4. Los políticos no se preocupan
\begin{tabular}{|l|l|c|r|r|c|}
\hline VÁlDOS S & & FRECUENCIA & PORCENTAE & PORCENTAE VÁLDO & PORCENTAEEACUMULADO \\
\hline & NS/NR & 21 & 5,5 & 5,5 & 5,5 \\
\hline & Muyen desacuerdo & 13 & 3,4 & 3,4 & 8,9 \\
\hline & Endesacuerdo & 45 & 11,7 & 11,8 & 20,7 \\
\hline & De acuerdo & 108 & 28,1 & 28,3 & 49,0 \\
\hline & Muyde acuerdo & 195 & 50,8 & 51,0 & 100,0 \\
\hline & Total & 382 & 99,5 & 100,0 & \\
\hline PERDIDOS & Sistema & 2 & 0,5 & & \\
\hline TOTAL & & 384 & 100,0 & & \\
\hline
\end{tabular}

Tabla 5. Compartir puntos de vista

\begin{tabular}{|c|l|r|r|r|r|}
\hline VÁlLDOS & & FRECUENCIA & PORCENTAE & PORCENTANE VÁLLD & PORCENTAE ACUMULADO \\
\hline & AMenudo & 49 & 12,8 & 12,8 & 12,8 \\
\hline & Algunas veces & 58 & 15,1 & 15,1 & 27,9 \\
\hline & Raramente & 152 & 39,6 & 39,6 & 67,4 \\
\hline & Nunca & 121 & 31,5 & 31,5 & 99,0 \\
\hline & No sabe & 1 & 0,3 & 0,3 & 99,2 \\
\hline & No responde & 3 & 0,8 & 0,8 & 100,0 \\
\hline & TOTAL & 384 & 100,0 & 100,0 & \\
\hline & & & & \\
\hline \multicolumn{7}{|l|}{} \\
\hline
\end{tabular}

ne que 238 de las 384 personas encuestadas, los de la población en general. Veamos la Taes decir, el $62 \%$ dice estar muy de acuerdo bla 6 .

con la afirmación, lo cual es un evidente $y$

claro reflejo de la gran desconfianza de la po- Sumando los porcentajes obtenidos en las blación por sus dirigentes políticos, haciendo respuestas "de acuerdo" y "muy de acuerdo" pensar que existe una deficiente ejecución y con la pregunta formulada, este porcentaaplicación de políticas de administración del je se sitúa en un $87,5 \%$ equivalente a 336 recurso, que lleva a la percepción en la po- personas de las 384 encuestadas, lo que inblación de que los dirigentes defienden solo dica en forma contundente que el ciudadasus intereses personales y no los de la pobla- no elector manifiesta que las personas que ción en general.

integran los estamentos de poder satisfacen en forma principal sus intereses personales, Este resultado evidencia una gran descon- muy por encima del postulado constituciofianza de la población por sus dirigentes po- nal de prevalencia de interés general y en líticos, llevando a la percepción de que ellos contravía del mandato impuesto por vía del defienden solo sus intereses personales y no voto.

Tabla 6. Quien esté en el poder busca sus intereses personales

\begin{tabular}{l|r|r|r|c|}
\hline \multicolumn{1}{|c|}{ VÁLLDOS } & FRECUENCIA & PORCENTAE & PORCENTAEVÁLIDO & PORCENTAEACUMULADO \\
\hline NS/NR & 17 & 4,4 & 4,4 & 4,4 \\
\hline Muyen desacuerdo & 7 & 1,8 & 1,8 & 6,3 \\
\hline Endesacuerdo & 23 & 6,0 & 6,0 & 12,2 \\
\hline De acuerdo & 98 & 25,5 & 25,5 & 37,8 \\
\hline Muyde acuerdo & 238 & 62,0 & 62,0 & 99,7 \\
\hline No sabe & 1 & 0,3 & 0,3 & 100,0 \\
\hline TOTAL & 384 & 100,0 & 100,0 & \\
\hline
\end{tabular}

REVVISTA VIS IURII I No. 3, Vol. 2185 - 109 I Enero-Junio, 2015 I Universidad Sergio Aiboleda I Esculala de Derecho I Seccional Santa Marta, Sede Centro 
En cuanto al grado de confianza en las insti- presarlo en la toma de decisión de elegir a tuciones, se consultó a los encuestados sobre un candidato.

entes incidentes en la política local. El análi-

sis hace referencia a un conjunto de opinio- En este sentido, se muestra que el elector nes sobre las principales instituciones que se cartagenero no cree en los partidos políticos. encargan de velar por el ejercicio democráti- El 79,7\% (187) de ellos tiene poca o ninguna co y la defensa de los intereses ciudadanos, confianza en estas instituciones, lo cual re-

según las competencias que la normatividad fleja que eventualmente no están siendo lo vigente les otorga. Las preguntas realizadas sobre la opinión estaban codificadas en un rango de 1 a 10 , el cual mide el grado de confianza que los ciudadanos manifiestan, en donde 1 denota "Ninguna confianza" y 10 indica "mucha confianza". suficientemente rigurosos o fuertes para generar cohesión y confianza en los ciudadanos que requieren atención de sus gobernantes, los cuales son precisamente elegidos por voto popular.

Tabla 7. Confianza en los partidos políticos

\begin{tabular}{|c|c|c|c|c|c|}
\hline VÁLIDOS & & FRECUENCIA & PORCENTAJE & PORCENTAUE VÁLIDO & PORCENTAJE ACUMULADO \\
\hline & Ninguna & 119 & 31,0 & 31,0 & 31,0 \\
\hline & Poca & 68 & 17,7 & 17,7 & 48,7 \\
\hline & Alguna & 106 & 27,6 & 27,6 & 76,3 \\
\hline & Mucha & 91 & 23,7 & 23,7 & 100,0 \\
\hline & Total & 384 & 100,0 & 100,0 & \\
\hline
\end{tabular}

En la tabla anterior, se muestra la confianza En cuanto al Concejo Distrital, el $81 \%$ de los que las personas tienen en los partidos po- ciudadanos encuestados manifiesta no telíticos tradicionales y nuevos, de tal forma nerle confianza a esta corporación, siendo que se pudiera establecer si en Cartagena los esta una institución que brinda las herrapartidos políticos representan instituciones mientas al Gobierno Distrital para adelantar fuertes, en las cuales los ciudadanos depo- sus políticas de gobierno. sitan su confianza ideológica para luego ex-

Tabla 8. Confianza en el Concejo Distrital

\begin{tabular}{|l|l|r|r|r|r|}
\hline VálLDOS & & FRECUENCIA & PORCENTAE & PORCENTAE VÁLIDO & PORCENTAE ACUMULADO \\
\hline & Ninguna & 99 & 25,8 & 25,8 & 25,8 \\
\hline & Poca & 113 & 29,4 & 29,4 & 55,2 \\
\hline & Alguna & 71 & 18,5 & 18,5 & 73,7 \\
\hline & Mucha & 101 & 26,3 & 26,3 & 100,0 \\
\hline & Total & 384 & 100,0 & 100,0 & \\
\hline
\end{tabular}

Las personas encuestadas manifiestan quela tores encuestados, la Personería Distrital, confianza que les genera las Juntas Adminis- entidad que vigila el cumplimiento de la nortradoras Locales se muestra muy deteriorada, ma, merece ninguna o poca confianza. Sin ya que un porcentaje equivalente al $79,4 \%$, es embargo, es válido reconocer que el porcendecir, 190 personas expresan tener ninguna o taje equivalente al $47,9 \%$, correspondiente a poca confianza en estas instituciones, lo que 184 personas encuestadas manifiestan tener refleja un alto grado de fragilidad institucio- confianza en esta entidad.

nal, teniendo en cuenta que estas desde el in-

terior de cada jurisdicción deben promover el Por su parte, en la Alcaldía Mayor la mayodesarrollo de los barrios y comunas y las que ría de las personas encuestadas equivalentes de primera mano han de conocer las necesi- al 55,2 \%, manifiesta tener ninguna o poca dades de los ciudadanos. Así se refleja en la confianza en esta entidad. Es importante sesiguiente tabla:

ñalar que en este aspecto, sumados los porcentajes de mucha con alguna confianza, no Para el 52,1\%, equivalente a 200 de los elec- alcanzan a superar los porcentajes sumados

Tabla 9. Confianza en la Junta Administradora Local

\begin{tabular}{|l|c|c|c|c|}
\hline \multicolumn{1}{|c|}{ VÁlLDOS } & FRECUENCIA & PORCENTAE & PORCENTAEVÁLIDO & PORCENTAEACUMULADO \\
\hline Ninguna & 115 & 29,9 & 29,9 & 29,9 \\
\hline Poca & 75 & 19,5 & 19,5 & 49,5 \\
\hline Alguna & 110 & 28,6 & 28,6 & 78,1 \\
\hline Mucha & 84 & 21,9 & 21,9 & 100,0 \\
\hline Total & 384 & 100,0 & 100,0 & \\
\hline
\end{tabular}

Tabla 10. Personería Distrita

\begin{tabular}{|c|c|c|c|c|}
\hline VÁLIDOS & FRECUENCIA & PORCENTAE & PORCENTAEVVÁLDDO & PORCENTAE ACUMULADO \\
\hline Ninguna & 88 & 22,9 & 22,9 & 22,9 \\
\hline Poca & 112 & 29,2 & 29,2 & 52,1 \\
\hline Alguna & 76 & 19,8 & 19,8 & 71,9 \\
\hline Mucha & 108 & 28,1 & 28,1 & 100,0 \\
\hline Total & 384 & 100,0 & 100,0 & \\
\hline
\end{tabular}

Tabla 11. Alcaldía Mayor

\begin{tabular}{|l|c|c|c|c|}
\hline \multicolumn{1}{|c|}{ VÁlLDOS } & FRECUENCIA & PORCENTAE & PORCENTAEV VÁLIDO & PORCENTAEACUMULADO \\
\hline Ninguna & 98 & 25,5 & 25,5 & 25,5 \\
\hline Poca & 114 & 29,7 & 29,7 & 55,2 \\
\hline Alguna & 69 & 18,0 & 18,0 & 73,2 \\
\hline Mucha & 103 & 26,8 & 26,8 & 100,0 \\
\hline Total & 384 & 100,0 & 100,0 & \\
\hline
\end{tabular}


de poca confianza con ninguna confianza, tal como puede observarse en la Tabla 11.

En cuanto a la Contraloría Distrital la opinión no le es tampoco favorable a la confianza que genera en el elector encuestado, ya que la tabla siguiente muestra que un $55,2 \%$ de ciudadanos, equivalentes a 212 personas, manifiesta tener ninguna o poca confianza en esta entidad, sin embargo contrasta el resultado obtenido de las personas que manifiestan tener "mucha" confianza en esta entidad, cuyo porcentaje equivale al $28,6 \%$.

(110 personas), frente al que expresa no tener "ninguna" confianza.

Al auscultar la opinión de los electores cartageneros sobre los medios de comunicación se refleja un porcentaje alto de favorabilidad, equivalente al $57,8 \%$ (222), ya que la mayoría de los encuestados manifestó tener

mucha confianza, frente a un menor porcentaje de poca confianza. Podría interpretars como un alto poder de los medios de comublación de carácter mediático hacia estos.

Otro de los aspectos de importancia para la investigación, era identificar el tipo de voto prevaleciente en la ciudad, para lo cual se consultó a los encuestados sobre el porque vota y si lo hace por convicción, preferencia por las propuestas o programas de gobierno de un candidato específico o cualquier otro factor. Las respuestas arrojaron resultados interesantes mostrados en la Tabla 14, en donde un $36 \%$ de los encuestados dice votar por convicción ideológica y un 23 \% lo hace porque le interesan los programas y propuestas que ofertan los candidatos.

Este resultado contrasta especialmente con

\begin{tabular}{|c|c|c|c|c|}
\hline VÁLDOS & FRECUENCIA & PORCENTAE & PORCENTAE VÁLDOO & PORCENTAJE ACUMULADO \\
\hline Ninguna & 106 & 27,6 & 27,6 & 27,6 \\
\hline Poca & 106 & 27,6 & 27,6 & 55,2 \\
\hline Alguna & 62 & 16,1 & 16,1 & 71,4 \\
\hline Mucha & 110 & 28,6 & 28,6 & 100,0 \\
\hline Total & 384 & 100,0 & 100,0 & \\
\hline
\end{tabular}

Tabla 13. Confianza en medios de comunicación

\begin{tabular}{|c|c|c|c|c|}
\hline VÁLIDOS & FRECUENCIA & PORCENTAE & PORCENTAUE VÁLLDO & PORCENTAJEACUMULADO \\
\hline Ninguna & 105 & 27,3 & 27,3 & 27,3 \\
\hline Poca & 57 & 14,8 & 14,8 & 42,2 \\
\hline Alguna & 104 & 27,1 & 27,1 & 69,3 \\
\hline Mucha & 118 & 30,7 & 30,7 & 100,0 \\
\hline Total & 384 & 100,0 & 100,0 & \\
\hline
\end{tabular}

el referente al $67,5 \%$ manifestado de su La pertenencia o militancia en un partido poco o ningún interés por la política, analiza- incentiva y favorece la participación electodo en la Tabla 3.

Tabla 14. Cuando usted vota

\begin{tabular}{|l|c|c|c|c|}
\hline \multicolumn{1}{|c|}{ VÁLIDOS } & FRECUENCIA & PORCENTAEE & PORCENTAEVÁLIDO & PORCENTAEACUMULADO \\
\hline N/A & 1 & 3 & 0,3 & 0,3 \\
\hline Convicción ideológica & 139 & 36,2 & 36,2 & 36,5 \\
\hline Preferencia por un candidato & 60 & 15,6 & 15,6 & 52,1 \\
\hline Propuestasy programas & 89 & 23,2 & 23,2 & 75,3 \\
\hline Compromiso pollitico & 69 & 18,0 & 18,0 & 93,2 \\
\hline Perteneceal partido del candidato & 20 & 5,2 & 5,2 & 98,4 \\
\hline Otro & 6 & 1,6 & 1,6 & 100,0 \\
\hline Total & 384 & 100,0 & 100,0 & \\
\hline
\end{tabular}

Fuente: Del autor obtenidas de encuestas aplicadas

Al consultarse sobre la pertenencia, participación o militancia en algún grupo o partido político, más del $50 \%$ de los encuestados manifestó no pertenecer a ninguno, marcando una fuerte tendencia hacia el desconocimiento, apatía o simplemente indiferencia por los procesos y asuntos políticos de la ciudad en general. Sin embargo, de los que sí opinaron pertenecer a algún partido, manifestaron su militancia en el Partido Liberal. Esto muestra la gráfica:

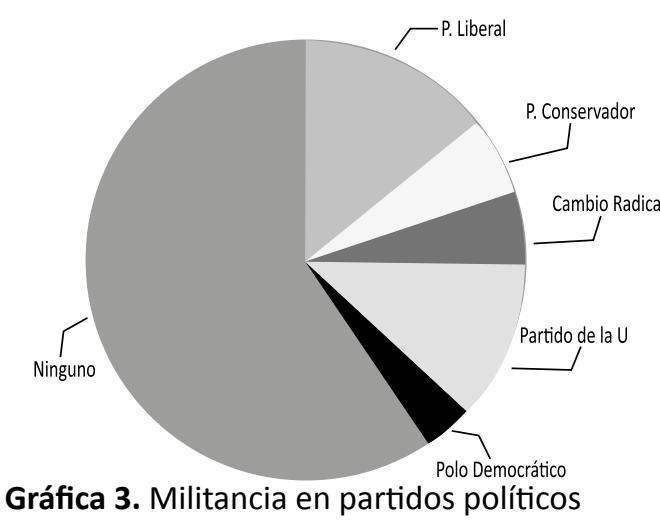

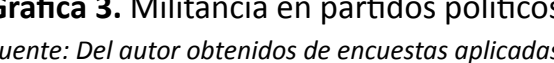

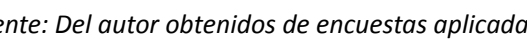

ral, debido a que estos se constituyen en los toral, aspecto de baja trascendencia en la ciudad, ya que a pesar de encontrarse reglamentados y constituidos los directorios, no se realizan actividades desde su interior, que estimulen o convoquen a su afiliación. entorno en la decisión del voto: una aproximación desde la teoría de la selección adversa

Con la finalidad de saber si el entorno influye o no en la decisión de voto de los encuestados, además de conocer si algunos factores influyen o no en el hecho de que este entorno influya sobre la decisión, se realizó el siguiente análisis de correlación basado en una regresión múltiple con dos variables explicativas. Cabe anotar que el modelo estimado no ha sido probado en cuanto a su violación de supuestos, dados los resultados principales agentes de reclutamiento elec-

\section{Los determinantes de la influencia del}


obtenidos, los cuales se muestran a continuación.

Variables introducidas/eliminadas

\begin{tabular}{|c|c|c|c|}
\hline Modelo & $\begin{array}{c}\text { Variables } \\
\text { introducidas }\end{array}$ & $\begin{array}{c}\text { Variables } \\
\text { eliminadas }\end{array}$ & Método \\
\hline 1 & $\begin{array}{c}\text { Votado Antes, } \\
\text { Partido }\end{array}$ & Introducir \\
\hline
\end{tabular}

Fuente: Del autor obtenidos de encuestas aplicadas

La tabla anterior resume el sistema de variables explicativas utilizadas, considerando que la variable explicada es precisamente la que hace referencia a si el entorno influye, o no, en la decisión de voto de los encuestados. Las explicativas por tanto, $y$ teniendo en cuenta los planteamientos de la teoría de selección adversa, se refieren a la participación previa en debates electorales y a la pertenencia o no en un partido político.

El entorno influye (variable dependiente)

Part: Participa en un partido

Vot: Ha votado antes

De acuerdo con la teoría de la selección adversa, lo deseable es que el individuo efectúe un análisis racional que permita una decisión de voto objetiva y consciente, además de que debe ser el resultado del ejercicio de su participación activa en grupos políticos de interés, movimientos o partidos. Por ello, se han escogido en particular estas dos variables, con la intención de poder mostrar el ni- variables.

Indicando que el entorno variará su influencia ante cambios en la pertenencia o no a un partido político y en el hecho de haber o no votado anteriormente. También se observ en la ecuación que el entorno depende de un componente autónomo, el cual es independiente del comportamiento de las demás

Tabla 15. Coeficientes

\begin{tabular}{|c|c|c|c|c|c|c|}
\hline Modelo & Coeficientes no estandarizados & Coeficientestipificados & $\mathbf{t}$ & Sig. & & \\
\hline & $\mathrm{B}$ & Errortí. & Beta & & & \\
\hline 1 & (Constante) & 3,126 &, 254 & & 12,298 &, 000 \\
\hline & Partido &, 036 &, 036 &, 050 &, 987 &, 324 \\
\hline & Votado Antes &,- 032 &, 148 & -011 & -214 &, 831 \\
\hline
\end{tabular}

a. Variable dependiente: Entorno Influye
Fuente: Del autor obtenidos de encuestas aplicadas
En este sentido, el modelo indica que si las te, los resultados del modelo de regresión, personas deciden aumentar su participación a la luz de la teoría de la selección adversa, en partidos políticos, aumentará de igual se pueden extraer algunos aspectos que a manera la influencia que el entorno puede tener sobre la decisión final de voto de un habitante cualquiera. Lo contrario ocurre con el haber votado con anterioridad, puesto que se puede observar que si aumenta la cantidad de veces en que la persona vota, se reducirá a futuro la influencia del entorno sobre su decisión de voto.

Sin embargo, los parámetros estimados deben ser usados solo para interpretaciones de relación y no para realizar proyecciones $n i$ inferencias, en la medida en que los parámetros resultan ser estadísticamente no significativos al nivel de confianza del $95 \%$ ya que la probabilidad del estadístico $t$ en cada caso, resulta ser mayor que el nivel de significancia del 0,05 .

Tabla 16. Resumen del modelo

\begin{tabular}{|c|c|c|c|c|}
\hline Modelo & $\mathbf{R}$ & $\begin{array}{c}\mathbf{R} \\
\text { cuadrado }\end{array}$ & $\begin{array}{c}\mathbf{R} \\
\text { cuadrado } \\
\text { corregida }\end{array}$ & $\begin{array}{c}\text { Error típ. de la } \\
\text { estimación }\end{array}$ \\
\hline 1 &, $052^{\mathrm{a}}$ &, 003 &,- 003 & 1,339 \\
\hline
\end{tabular}

Fuente: Del autor obtenidos de encuestas aplicadas

Además de ello, el modelo tiene un ajuste global o nivel de explicación de las variables con respecto a la dependiente, de un 5,2\%, porcentaje que se considera sumamente bajo para corroborar que el modelo resulta ser consistente.

\section{A MODO DE CONCLUSIÓN}

Con base en el análisis anteriormente referenciado y teniendo en cuenta, especialmenmodo de conclusiones permiten entender de manera menos tecnicista los resultados obtenidos y la relación entre las variables anteriormente descritas.

Se obtuvo que la mayoría de personas inscritas en el censo electoral son de edades de 40 años o más y que poseen niveles de escolaridad de secundaria completa. Ello marca en primera instancia, en la interpretación de la teoría, que comienzan a aparecer perfiles característicos que pueden ser comparados con lo propuesto.

Por ejemplo, para lograr una decisión objetiva según la teoría, es necesario conocer a cabalidad el entorno y los factores propios de cada alternativa, además de que debe poseerse un nivel de conocimiento asertivo, que es producto de la escolaridad y la educación recibida a lo largo de la vida. Los datos muestran que efectivamente la ciudad de Cartagena tiene un censo electoral nutrido de personas con el conocimiento por lo menos básico para enfrentar procesos electorales con eficiencia.

Sin embargo, otros factores también influyen y no precisamente tienen que ver con la escolaridad. Uno de ellos, está relacionado con el interés en la política. Los resultados mostraron que solo un $30 \%$ de la población encuestada dice interesarse por los procesos políticos, contrastando con el $70 \%$ que opi- 
Ello sin duda ofrece un panorama más claro donde empiezan a aparecer elementos seminales propios de una decisión ocasionada por una selección adversa, en tanto a que si la mayoría de la gente muestra desinterés por la política, es probable que sus decisiones estén basadas en factores diferentes a la racionalidad y la objetividad que conlleva el debate electoral.

Además de ello, se observó una alta prevalencia de personas que no confían en algunas instituciones estructurantes de la democracia representativa, como los Partidos Políticos, el Concejo, la Personería, la Alcaldía la Gobernación e incluso la Iglesia, las cuales gozan de un nivel de percepción negativa en la población que hace parte del censo electoral, que también muestra una alta orientación por parte de la población civil a ejerce el voto de manera no consciente o por lo menos indiferente de las propuestas y potencialidades de los aspirantes.

Por último, el modelo dio como resultado e hecho de que posiblemente a mayor cantidad de personas participando en los partidos, se podría modificar el esquema tradiciona electoral y por tanto, hacer que el entorno sea el principal influyente de la decisión final de voto y no los factores que distorsionan su objetividad y que permita que se incurra en un comportamiento de selección adversa, donde se elige a los gobiernos de más mala calidad, porque simplemente existe o prevalece un desinterés generalizado por los procesos politicos locales, tal y como lo sugiere la teoría.

\section{REFERENCIAS}

Akerlof, G. (1970). Market of Lemons: Quality Uncertainty and the Market Mechanism. Quarterly Journal of Economics, 87.

Alcántara, M. \& García, F. (2008). Eleccione y política en América Latina. FEEM.

Anduiza, E. \& Bosch, A. (2004). Corportamiento político y electoral. Barcelona: Arie Ciencia Política.

Campbell, A., Converse, E., Miller, W. \& Stokes, D. (1960). The American Voter. New York: Wiley.

Carmines, E. \& Huckeld, R. (1996). "Political Behavior: An Overview". In R. Goodin \& H.-D. Klingeman (Eds.), A New Handbook of Political Science. New York: Oxford University Press.

Cartagena Cómo Vamos [CCV] (2010). Clima de opinión y calidad de vida. Cartagena de Indias: Encuesta de percepción ciudadana.

Cátedra Konrad Adenauer de Comunicación y Democracia (2002). Medios y próceso electoral en Colombia. Bogotá, D.C.: Fundación Konrad Adenauer. Pontificia Universidad Javeriana.

Converse, P. \& Markus, G. (1979). Plus ça change...: The New CPS Election Study Panel. American Political Science Review, 73(01) $32-49$
Dalh, R. (1971). Polyarchy: Participation and Opposition. New Haven: Yale University Press. cepciones básicas en economía de la información y la selección adversa en el objeto de estudio de la teoría organizacional. Revista Downs, A. (1957). An Economic Theory of De- Economía y Desarrollo, 4(1). mocracy. New York: Harper and Row.

Morris P., F. (1985). Retrospective Voting in Gaitán, P. (1989). "La elección popular de Al- American Elections. New Haven: Yale Univercaldes: Un desafío para la democracia". Aná- sity Press. lisis Político, 3. Colombia Elecciones 2000. Ediciones Académicas.

Indias. Revista Panorama Económico, (13).
Departamento de Investigaciones Económ

cas y Sociales. Universidad de Cartagena.

Guzmán, C. (2005). Política, descentralización y subsistemas regionales de partidos en Colombia 1988-2000. Ibagué: Universidad de bagué.

Huckfeldt, R. (1983). Social contexts, socia networks, and urban neighborhoods: Environmental constraints on friendship choice. American Journal of Sociology, 89(3), 651 669

Orgulloso, A. (2005). Cartagena sitiada: entre la pobreza y la corrupción. Bogotá.

Santana, J. (2007). Los sistemas políticos contemporáneos. Documento de Internet. Consultado el 02 de marzo de 2012

Science of the University of the Andes and CEREC.

Kinder, D. \& Kiewiet, R. (1981). "Sociotropi Politcs: The American Case". British Journal of Political Science, 11.

Simon, H. (1957 y 1985). Reasoning and choice: Explorations in political psychology. Cambridge University Press.

Lazarsfeld, B., Berelson, B. \& Gaudet, H. Sniderman, P., Brody, B. \& Tetlock, P. (1991). (1944). The people Choice. New York: Duell, Reasoning and Choice: Explorations in PolitiSloan \& Pearce. cal Psychology. New York: Cambridge Univerparticipation: how and why do people get involved in politics. Chicago: Rand McNally. 